\title{
Binary Orbits from Interferometric Data
}

\author{
C. A. HUMMEL \& J. T. ARMSTRONG \\ NRL/USNO OIP and USRA, U.S. Naval Observatory, 34th St. \& \\ Massachusetts Ave. NW, Washington, DC 20390, USA
}

\section{INTRODUCTION}

Using the variable baseline of the Mark III Optical Interferometer (Shao et al. 1988) with lengths ranging from 3 to $31.5 \mathrm{~m}$, we measured the fringe visibility of stars in three $25 \mathrm{~nm}$ bandpass filters, at wavelengths between $800 \mathrm{~nm}$ and 450 $\mathrm{nm}$. This setup gives fringe spacings ranging from 55 mas to 3 mas.

255 stars brighter than fifth magnitude and north of $-10^{\circ}$ declination were selected from the Batten et al. (1989) catalog of spectroscopic binaries. We compiled values for the orbital axes from the McAlister \& Hartkopf (1988) catalog. If unknown (in the majority of cases), they were estimated from the masses predicted from the spectral types, the periods (if known), and the distances to the binaries. Priority was given to the binaries with orbital axes between 3 mas and 40 mas. Observational procedures and the calibration of the data are described by Armstrong et al. (1992). Statistical and calibration uncertainties are in the range of $1 \%$ (red channel) to $10 \%$ (blue channel).

\section{THE DATA BASE}

For about 130 stars we have accumulated sufficient data since 1988 to reach a preliminary judgement as to whether the visibility shows obvious (larger than $20 \%$ ) variations on any of the observed baselines, indicative of multiple structure. As expected, priority stars have the highest detection rate: nearly half of the stars show variations larger than those of a 2.4 magnitude difference system with unresolved components. Since under unfortunate circumstances (e.g., observations with the wrong baseline of a highly elliptical orbit at periastron) variations could perhaps be missed, these statistics should be considered as a guideline. Five final orbits have been determined.

\section{FINDING ORBITS}

Finding an orbit begins with the determination of $(\rho, \theta)$ values corresponding to the mean positions of the binary during the nights (for details, we refer to the paper by Armstrong et al. (1992). We note that the use of three unevenly spaced wavelengths resolves the ambiguity of different possible $(\rho, \theta)$ combinations in nearly all cases (due to the lack of phase information, however, the position angle is subject to a $180^{\circ}$ ambiguity). After obtaining $(\rho, \theta)$ values corresponding to a significant phase coverage of the orbit, a preliminary orbit can be fitted to the data. The orbital period often is known from the spectroscopic observations to a much higher degree of precision than can be achieved by our observations. 


\section{IMPROVING THE ORBIT BY SOLVING FOR THE COMPLETE MODEL}

By fitting the 7 orbital and 6 system elements (i.e. the diameters and magnitude differences of the stars at the three wavelengths) directly to the observed visibilities, uncertainties in the parameters can be decreased for the following reasons: (1) data of nights which are not sufficient to constrain a single night's model can be used, (2) the system parameters are constrained by all data at the same time, and (3) the method also allows for significant orbital motion in the course of the night if the period is short and/or the binary is close to periastron. The Levenberg-Marquardt algorithm converges normally within 15 iterations from the initial model.

In the following figures we present preliminary orbits of 4 more binaries. Each plot gives the model parameter values and model orbit, the total number of visibilities in the three channels and the reduced $\chi^{2}$ of the fit to the visibilities. The major axis of the ellipse is a line labeled with the letter $T$ at the periastron passage. The apparent velocity of the orbital motion can be derived from the separation of the dots, which is $P / 200$; the direction is indicated by a little arrow. In order to visualize constraints imposed by the data of a single night (i) on the orbit, we minimized $\chi_{i}^{2}$ by varying the model position $\left(r_{i}, \theta_{i}\right)$ and calculated an error ellipse corresponding to an increase of the reduced $\chi^{2}$ by a factor of $1+1 / n$, where $n$ is the number of independent data points of night $(i)$.

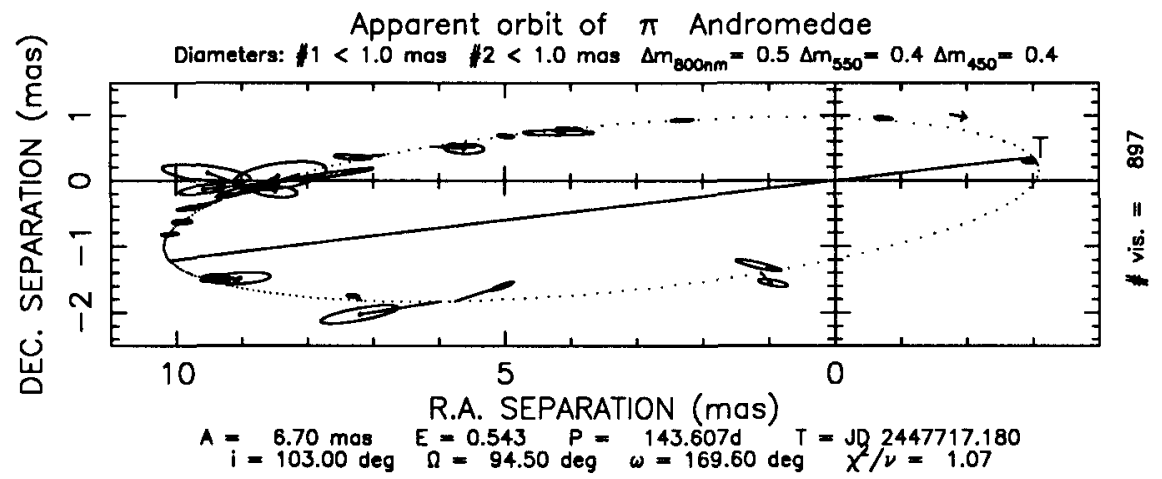

\section{REFERENCES}

Armstrong, J.T., Mozurkewich, D., Vivekanand, M., Simon, R.S., Johnston, K.J., Pan, X.-P., Shao, M., \& Colavita, M.M. 1992, AJ, 104, 241

Batten, A.H., Fletcher, J.M., \& MacCarthy, D.G. 1989, Eighth Catalogue of the Orbital Elements of Spectroscopic Binary Systems, Publ. Dominion Astrophys. Obs., 17

McAlister, H.A. \& Hartkopf, W.I. 1988, Second Catalog of Interferometric Measurements of Binary Stars, CHARA Contrib. No. 2

Shao, M., Colavita, M.M., Hines, B.E., Staelin, D.H., Hutter, D.J., Johnston, K.J., Mozurkewich, D., Simon, R.S., Hershey, J.L., Hughes, J.A., \& Kaplan, G.H. 1988, $A \& A, 193,357$ 

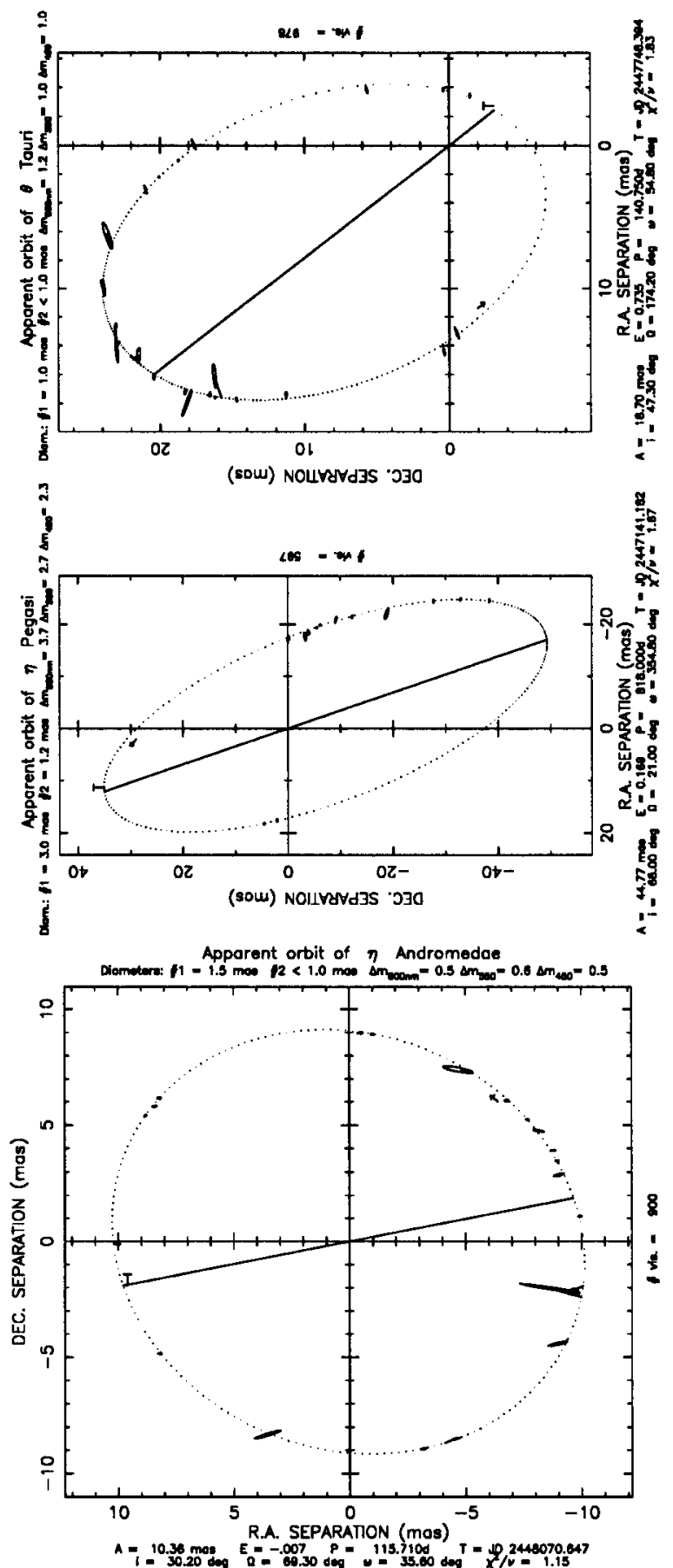